\title{
A study of factors related to asthma exacerbation using a questionnaire survey in Niigata Prefecture, Japan
}

\author{
Hiroshi Ueno, ${ }^{1}$ Toshiyuki Koya, ${ }^{1}$ Takashi Hasegawa, ${ }^{2}$ Masachika Hayashi, ${ }^{1}$ Kazutaka Yoshizawa, ${ }^{1}$ \\ Eiichi Suzuki, ${ }^{2}$ Toshiaki Kikuchi, ${ }^{1}$ the Niigata Asthma Treatment Study Group
}

\begin{abstract}
Background: As indices of asthma control, exacerbations are equally important with symptoms and respiratory function. Thus, it is critical to recognize the risk factors of exacerbation.

Objective: We conducted a questionnaire survey of asthma patients in Niigata Prefecture to clarify the factors involved in asthma exacerbation.

Methods: The questionnaire survey was carried out in patients and their physicians from September to October 2014. In 2015, the same sample population also received a questionnaire about current asthma control and exacerbation.

Results: One hundred patients experienced asthma exacerbation during the 1-year period. There were significant differences in age, sex, history of hospitalization due to asthma, smoking history, Asthma Control Test, treatment step, and transient steroid treatment history in the previous year between the exacerbation group and non-exacerbation group. On multivariate analysis, there was a significant difference in history of transient steroid therapy, history of hospitalization associated with asthma attacks, and nonsmoking history. Cluster analysis of cases with exacerbation was classified into three clusters. Cluster 1 comprised slightly older cases with smoking history, Cluster 2 had more females, non-smoking and nonatopic cases with uncontrolled symptoms, and Cluster 3 had more females, non-smoking and mild atopic cases.
\end{abstract}

Conclusions: Our findings suggest that patients with asthma exacerbation in the previous year and nonsmoking females are important targets for the study of asthma exacerbation. The adequate treatment of women patients might be important for the prevention of asthma exacerbation.

Key words: Bronchial asthma, exacerbation, questionnaire survey, nonsmoking, menopause

\footnotetext{
From:

${ }^{1}$ Department of Respiratory Medicine and Infectious Diseases, Niigata University Graduate School of Medical and Dental Sciences, Niigata City, Niigata, Japan.

2 Department of General Medicine, Niigata University Medical and Dental Hospital, Niigata City, Niigata, Japan.
}

\author{
Corresponding author: \\ Toshiyuki Koya \\ Department of Respiratory Medicine and Infectious Diseases, Niigata \\ University Graduate School of Medical and Dental Sciences, 1-757 \\ Asahimachi-Dori, Chuo-ku, Niigata City, Niigata 951-8510, Japan \\ E-mail: tkoya@med.niigata-u.ac.jp
}

\section{Introduction}

Bronchial asthma is characterized by symptoms such as wheezing, chest discomfort, shortness of breath, and cough based on chronic airway inflammation and reversible airway obstruction. ${ }^{1}$ In guidelines such as the Global Initiative for Asthma (GINA) and the National Asthma Education and Prevention Program (NAEPP), two asthma treatment goals are proposed, ${ }^{2,3}$ to achieve asthma control and to reduce future risk of asthma. Reduction of future risk includes the maintenance of respiratory function and prevention of acute exacerbation and asthma death. Acute exacerbations are a major cause of asthma death, and the American Thoracic Society and European Respiratory Society have reported that acute exacerbations are related to severe stress and anxiety and higher medical expenses. ${ }^{4}$ Therefore, the prevention of asthma exacerbation is an important goal for asthma treatment.

Medical circumstances such as national public medical insurance and the unique culture of Japan may influence asthma treatment. For example, rather than monotherapy with inhaled corticosteroid (ICS), a combination therapy of ICS and long-acting beta-agonist is mainly used for asthma control. 
Other circumstances include unique lifestyle such as using futons that allow mites to thrive easily and humid climate. Based on these, it is difficult to compare the risk factors of asthma exacerbation in Japan with those of other countries. In the analysis of risk of acute exacerbation in the Japanese population, Sato et al. reported that patient-based questionnaires and forced expiratory volume in 1 second $\left(\mathrm{FEV}_{1}\right)$ are indicators for risk of future exacerbation. ${ }^{5}$ Meanwhile, Tanaka et al. reported that the worsening of symptoms in the previous year is a predictor of future exacerbation, ${ }^{6}$ consistent with previous reports. ${ }^{7}$ However, the samples were collected from limited facilities, and overall, both findings are inconsistent.

The Niigata Asthma Treatment Study Group (NATSG) was established in 1997 and is engaged in exchanging asthma treatment information, improving asthma control, and preventing asthma deaths in Niigata Prefecture. The main activities of the NATSG include conducting questionnaire surveys of asthma cases in the prefecture; these were started in 1998 and are conducted every year or every alternate year. Based on this questionnaire survey data, many studies have been conducted on the situation and features of asthma patients in Niigata prefecture, Japan. ${ }^{8-11}$

In this study, we conducted a questionnaire survey between 2014 and 2015 in Niigata Prefecture and analyzed the factors involved in asthma exacerbation in the Japanese population.

\section{Methods \\ Subjects}

The questionnaire survey was performed from September to October both in 2014 and 2015, in accordance with the ethical principles for medical research involving human subjects and the Declaration of Helsinki and with approval from the ethics committee of Niigata University (approval no. 1231). The subjects included patients who were diagnosed with asthma according to the guidelines of the Japanese Society of Allergology. The institutions involved were 28 large hospitals $(\geq$ 200 beds), 15 small hospitals ( $<200$ beds), and 69 clinics (no beds). Among the patients who participated in both the 2014 and 2015 questionnaire surveys, we enrolled patients who reported the latest data for respiratory function test in the 2014 questionnaire and a description of asthma exacerbation in the 2015 questionnaire. A total of 552 patients were therefore enrolled in this study.

\section{Protocol}

After providing informed consent, the patients completed a questionnaire on age, sex, smoking status, disease duration, Adherence Starts with Knowledge-12 (ASK-12), and Asthma Control Test (ACT). Their physicians also provided data on the contents of therapy, recent pulmonary function test, and existence of exacerbation. Asthma exacerbation was defined as systemic steroid use (or increased use) for $\geq 3$ days due to asthma exacerbation, hospitalization due to worsening of asthma, emergency outpatient visit for asthma exacerbation, or unscheduled consultation due to asthma exacerbation.

\section{Statistical analysis}

The results were expressed as medians $\left(25^{\text {th }}-75^{\text {th }}\right.$ interquartile range), because the data for continuous variables were mainly distributed non-normally. To compare differences among the groups, one-way analysis of variance and Bonferroni's multiple comparison tests were used. The in-group comparisons were performed using Wilcoxon's signed-rank test. Comparisons for all pairs were performed using the Kruskal-Wallis test. Multivariate analysis was used to identify the variables that influenced asthma control. Variables that were statistically significant in the dichotomous analysis were applied in the multivariable logistic regression analysis. We performed a hierarchical cluster analysis on the exacerbation group using Ward's method, as reported previously. ${ }^{12,13}$ In brief, we chose important variables for cluster establishment, including disease duration, sex, age, disease type, smoking status, ACT score, medication content, and $\% \mathrm{FEV}_{1}$. Decision tree analysis was conducted to retrieve methods of clinical categorization for ease of usage. All of these factors were modified to the nominal scale and input. Most statistical analyses were performed using JMP software version 11 (SAS Institute, Inc., Tokyo, Japan). Decision tree analysis was performed using CAnalysis ver. 4.0 software. For the adjustment of age and sex, optimal matching using EZR (Saitama Medical Center, Jichi Medical University, Saitama, Japan) was performed. For all statistical analyses, $\mathrm{p}<0.05$ was considered significant.

\section{Results}

A total of 100 patients were included in the group that experienced at least one episode of exacerbation during the 1 -year period (exacerbation group). Compared with the non-exacerbation group, the exacerbation group were younger, women-dominant, and nonsmoker-dominant. Regarding treatment step defined by the Japanese Society of Allergology, the prevalence of patients with step 4 (i.e., most intensive treatment) was significantly higher in the exacerbation group. Moreover, administration of other maintenance therapies such as leukotriene receptor antagonist, long-acting muscarinic receptor antagonist, oral sustained-release theophylline, and oral corticosteroid (OCS) was significantly higher in the exacerbation group. Furthermore, ACT score was significantly lower in the exacerbation group. In terms of comorbidity, the prevalence of allergic rhinitis and osteoporosis was higher in the exacerbation group (Table $\mathbf{1}$ ).

Table 1. Comparison of clinical characteristics between subjects with and without exacerbation

\begin{tabular}{lccc|} 
& $\begin{array}{c}\text { Exacerbation } \\
(+)\end{array}$ & $\begin{array}{c}\text { Exacerbation } \\
(-)\end{array}$ & p-value \\
\hline No. of cases $(\mathrm{n})$ & 100 & 452 & \\
\hline Age (years), median (IQR) & $57[43-71]$ & $63[50-72]$ & 0.04 \\
\hline Female, n (\%) & $70(70)$ & $255(56.4)$ & 0.01 \\
$\begin{array}{lccc}\text { BMI }\left(\mathrm{kg} / \mathrm{m}^{2}\right), \text { median } \\
\text { (IQR) }\end{array}$ & $\begin{array}{c}23.2 \\
{[20.2-25.5]}\end{array}$ & $\begin{array}{c}23.0 \\
{[20.7-25.3]}\end{array}$ & 0.72 \\
\hline
\end{tabular}

BMI: body mass index, IQR: interquartile range 
Table 1. (Continued)

\begin{tabular}{lccc} 
& $\begin{array}{c}\text { Exacerbation } \\
(+)\end{array}$ & $\begin{array}{c}\text { Exacerbation } \\
(-)\end{array}$ & p-value \\
\hline Duration of disease (years) & $10[4-20]$ & $9[4-28]$ & 0.62 \\
\hline $\begin{array}{l}\text { Age of onset (years), } \\
\text { median (IQR) }\end{array}$ & $43[31-57]$ & $59[35-63]$ & 0.03 \\
\hline $\begin{array}{l}\text { Smoking history } \\
\text { (non/ex/cur), n (\%) }\end{array}$ & $\begin{array}{c}68 / 27 / 5 \\
68 / 27 / 5)\end{array}$ & $\begin{array}{c}230 / 185 / 36 \\
(51 / 41 / 8)\end{array}$ & 0.009 \\
\hline Atopic disease type, n (\%) & $60(60)$ & $286(63.2)$ & 0.57 \\
\hline $\begin{array}{l}\text { Immunoglobulin E } \\
\text { (IU/ml), median (IQR) }\end{array}$ & $167[50-620]$ & $158[52-442]$ & 0.44 \\
\hline \%FEV (\%), median (IQR) & $93[76-110]$ & $95[83-108]$ & 0.50 \\
\hline Treatment step 4, n (\%) & $32(32)$ & $53(12)$ & $<0.0001$ \\
\hline Asthma Control Test, & $22[19-24]$ & $24[21-24]$ & $<0.0001$ \\
median (IQR) & & & \\
\hline LTRA use, n (\%) & $68(68)$ & $209(46)$ & $<0.0001$ \\
\hline LAMA use, n (\%) & $11(11)$ & $21(4.6)$ & 0.0003 \\
\hline OSRT use, n (\%) & $40(40)$ & $99(22)$ & 0.0003 \\
\hline OCS use, n (\%) & $12(12)$ & $17(3.8)$ & 0.002 \\
\hline Allergic rhinitis, n (\%) & $43(43)$ & $45(34.4)$ & 0.039 \\
\hline GERD, n (\%) & $11(11)$ & $43(9.9)$ & 0.65 \\
\hline Osteoporosis, n (\%) & $7(7)$ & $9(2)$ & 0.001 \\
\hline
\end{tabular}

IQR: interquartile range, LTRA: leukotriene receptor antagonist, LAMA: longacting muscarinic receptor antagonist, non/ex/cur: nonsmoker/ex-smoker/ current smoker, OSRT: oral sustained-release theophylline, OCS: oral corticosteroid, GERD: gastroesophageal reflux disease. Data expressed in medians (IQR).

The exacerbation group had higher prevalence with regard to history of hospitalization than the exacerbation group. The prevalence of attack episodes and OCS burst episodes in the previous year was also significantly higher in the exacerbation group (Table 2).

Table 2. Comparison of past episodes related with exacerbation between subjects with and without exacerbation

\begin{tabular}{|c|c|c|c|}
\hline & $\begin{array}{c}\text { Exacerbation } \\
(+)\end{array}$ & $\begin{array}{c}\text { Exacerbation } \\
(-)\end{array}$ & p-value \\
\hline $\begin{array}{l}\text { History of hospitalization, } \\
\mathrm{n}(\%)\end{array}$ & 39 (39) & $93(21)$ & 0.0002 \\
\hline $\begin{array}{l}\text { Attack episodes in the } \\
\text { previous year, } \mathrm{n}(\%)\end{array}$ & $56(56)$ & $118(26)$ & $<0.0001$ \\
\hline $\begin{array}{l}\text { OCS burst episode in the } \\
\text { previous year, n (\%) }\end{array}$ & $50(50)$ & 45 (10) & $<0.0001$ \\
\hline $\begin{array}{l}\text { Emergency department } \\
\text { use, n (\%) }\end{array}$ & $32(32)$ & $102(22.6)$ & 0.0519 \\
\hline
\end{tabular}

OCS: oral corticosteroid.
To determine the factors associated with asthma exacerbation, multivariate analysis was conducted. Based on data from the comparison test between the exacerbation and non-exacerbation groups, age, OCS burst, smoking history, sex, ACT score, allergic rhinitis, treatment step, and osteoporosis were applied as variables. In the multivariate analysis, significant differences were observed in OCS burst in the previous year, history of hospitalization, and smoking history. Interestingly, a nonsmoking history was more likely to cause exacerbation (Table 3).

Table 3. Multiple regression analysis of factors related to asthma exacerbation

\begin{tabular}{|lccc}
\multicolumn{1}{c}{ Factor } & $\begin{array}{c}\text { Hazard } \\
\text { ratio }\end{array}$ & 95\% CI & p-value \\
\hline Age ( $\leq$ 64 years) & 1.36 & $0.78-2.39$ & 0.268 \\
OCS burst (yes) & 6.01 & $3.28-11.1$ & $<0.001$ \\
\hline Smoking history (never) & 2.57 & $1.39-4.90$ & 0.003 \\
\hline History of hospitalization (yes) & 2.31 & $1.32-4.02$ & 0.002 \\
\hline Female & 0.97 & $0.52-1.82$ & 0.93 \\
\hline Asthma Control Test score (<22) & 1.46 & $0.86-2.47$ & 0.15 \\
\hline Allergic rhinitis (yes) & 1.26 & $0.92-2.17$ & 0.40 \\
\hline Treatment step (4) & 1.82 & $0.93-3.51$ & 0.07 \\
\hline Osteoporosis (yes) & 1.10 & $0.29-3.98$ & 0.87 \\
\hline
\end{tabular}

OCS: oral corticosteroid.

Based on the multivariate analysis, cluster analysis was performed to further analyze the cause of exacerbation (Table 4). The subjects were classified into three groups: male-dominant group with slightly older age, smoking history, and poor respiratory function and asthma control (Cluster 1); female-dominant group with nonatopic disease type with relatively poor asthma control (Cluster 2); female-dominant group with better asthma control and mild atopic disease type (Cluster 3). Both Clusters 2 and 3 were mainly nonsmokers with good respiratory function.

Table 4. Demographic features of the groups by cluster analysis.

\begin{tabular}{lcccc} 
& Cluster 1 & Cluster 2 & Cluster 3 & p value \\
Sample & 40 & 41 & 17 & \\
Sex (M/F) & $19 / 21$ & $8 / 33$ & $1 / 16$ & 0.0015 \\
& 60.0 & 58.7 & 49.9 & \\
Age & {$[55.34-64.5]$} & {$[54.2-63.3]$} & {$[42.9-57.0]$} & 0.055 \\
Onset of & 39.2 & 50.1 & 41.9 & \multirow{2}{*}{0.018} \\
Disease & {$[33.7-44.6]$} & {$[44.7-55.6]$} & {$[33.5-50.3]$} & \\
BMI & 23.2 & 23.9 & 23.4 & \multirow{2}{*}{0.82} \\
& {$[21.9-24.7]$} & {$[22.5-25.2]$} & {$[21.3-25.5]$} & \\
\hline
\end{tabular}


Table 4. (Continued)

\begin{tabular}{|c|c|c|c|c|}
\hline & Cluster 1 & Cluster 2 & Cluster 3 & p value \\
\hline $\begin{array}{l}\text { Duration of } \\
\text { disease }\end{array}$ & $\begin{array}{c}20.8 \\
{[17.5-24.1]}\end{array}$ & $\begin{array}{c}8.6 \\
{[5.4-11.9]}\end{array}$ & $\begin{array}{c}8.0 \\
{[2.9-13.0]}\end{array}$ & $<0.001$ \\
\hline Atopic (+/-) & $33 / 7$ & $13 / 28$ & $14 / 3$ & $<0.001$ \\
\hline $\begin{array}{l}\text { Smoking } \\
\text { history } \\
\text { (current or } \\
\text { past/never) }\end{array}$ & $24 / 16$ & $4 / 37$ & $3 / 14$ & $<0.001$ \\
\hline $\begin{array}{l}\text { Attack in previ- } \\
\text { ous year }(+/-)\end{array}$ & $26 / 13$ & $22 / 14$ & $7 / 10$ & 0.19 \\
\hline ACT & $\begin{array}{c}21.6 \\
{[20.7-22.5]}\end{array}$ & $\begin{array}{c}19.5 \\
{[18.56-20.4]}\end{array}$ & $\begin{array}{c}24.0 \\
{[22.6-25.4]}\end{array}$ & $<0.001$ \\
\hline $\begin{array}{l}\text { Treatment step } \\
(1-2 / 3-4)\end{array}$ & $7 / 33$ & $10 / 31$ & $14 / 3$ & $<0.001$ \\
\hline$\% \mathrm{FEV}_{1}$ & $\begin{array}{c}73.9 \\
{[67.8-80.1]}\end{array}$ & $\begin{array}{c}103.6 \\
{[97.6-109.2]}\end{array}$ & $\begin{array}{c}99.5 \\
{[90.0-108.9]}\end{array}$ & $<0.001$ \\
\hline
\end{tabular}

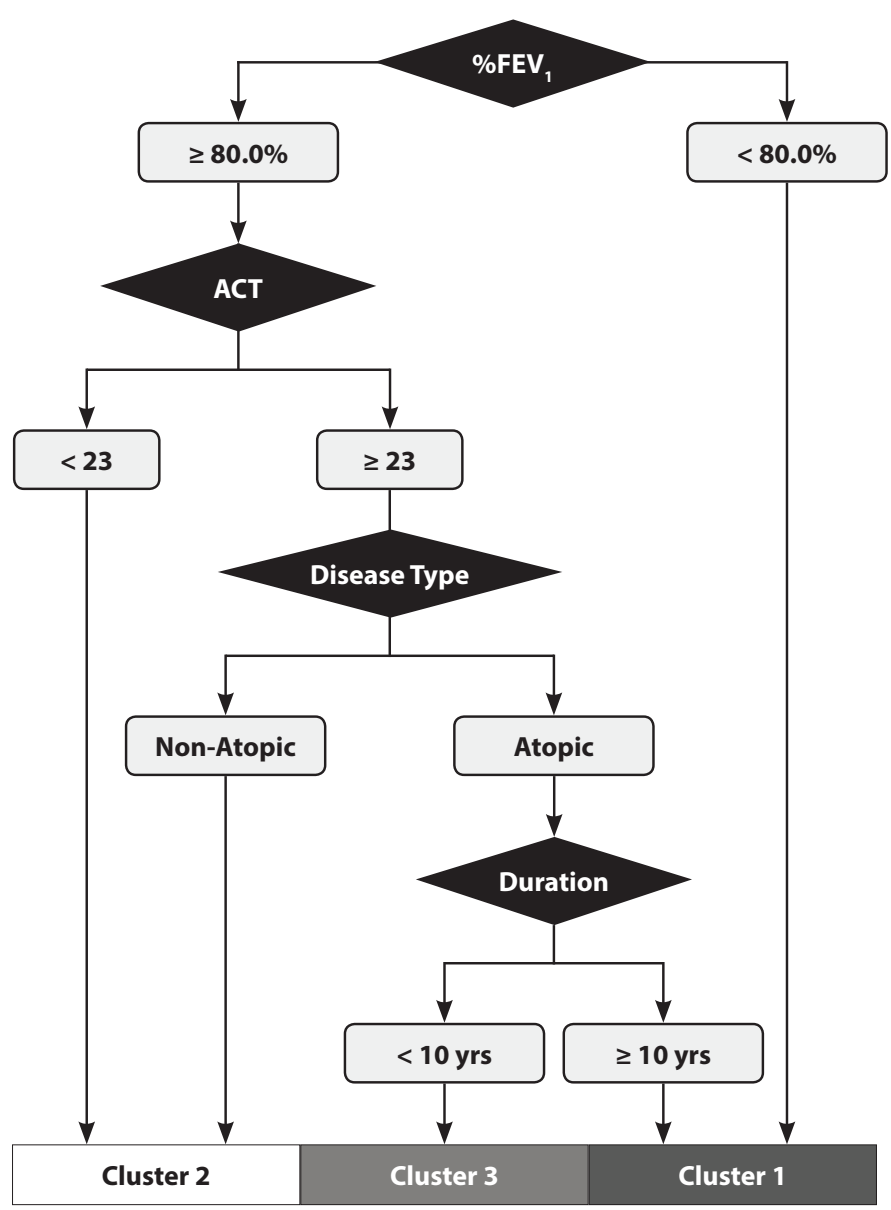

Figure 1. Decision tree analysis. The patients are classified into three clusters based on $\% \mathrm{FEV}_{1}$, ACT score, disease type, and disease duration.

$\% \mathrm{FEV}_{1}$, percent predicted forced expiratory volume in 1 second; ACT, Asthma Control Test
Table 5. Sensitivity and specificity of categorization in different clusters

\begin{tabular}{lccc} 
& Cluster 1 & Cluster 2 & Cluster 3 \\
\hline Sensitivity & $95.0 \%$ & $92.7 \%$ & $58.8 \%$ \\
Specificity & $87.9 \%$ & $88.1 \%$ & $100.0 \%$ \\
\hline
\end{tabular}

From the valuable parameters, we further retrieved 4 parameters including $\% \mathrm{FEV}_{1}$, ACT score, disease type (atopic or non-atopic), and disease duration to establish an easily used method of clinical categorization using discriminant analysis and decision tree analysis. The clusters were differentiated using these 4 parameters with a sensitivity of $95.0 \%, 92.7 \%$, and $58.8 \%$ and a specificity of $87.9 \%, 88.1 \%$, and $100 \%$ in cluster 1, 2, and 3, respectively (Figure 1 and Table 5).

\section{Discussion}

Asthma exacerbation causes aggravation of acute/subacute symptoms and a decline in respiratory function, impairing the patient's quality of life and being a major cause of burden on treatment costs. Although its prevention is important, patients with good current control do not necessarily escape exacerbation. A previous report described that the risk of future exacerbation is caused by a mechanism different from the current control state. ${ }^{14}$ To elucidate factors related to asthma exacerbation in the Japanese population, we analyzed factors of the same patients who underwent a questionnaire survey for 2 consecutive years.

To determine the classification of patients who had exacerbation, we performed a cluster analysis dividing the patients into three clusters. Cluster 1 included patients with smoking history, higher treatment steps, and lower respiratory function. Kupczyk M et al. reported that smoking history and a high fraction of exhaled nitric oxide (FeNO) are risk factors of asthma exacerbation, ${ }^{15}$ consistent with the results of our study. The other two clusters were women-dominant and nonsmoker-dominant. Cluster 2 comprised nonatopic cases with poor asthma control despite higher treatment steps, while Cluster 3 included atopic cases with good asthma control and lower treatment steps. FEV, values were good in both clusters. However, it has been often reported that women have a higher prevalence of hospitalizations, rescue consultations, and unscheduled consultations despite their good respiratory function and oxygenation. ${ }^{16,17}$ Thus, adequate asthma management is required for female patients to prevent exacerbation despite treatment.

Cluster 2 comprised many patients with poor control of asthma symptoms despite higher treatment. Compared with Cluster 3, Cluster 2 showed a significantly higher age of onset and a tendency of higher overall age. Although both clusters were female-dominant, we focused on menopausal age as a difference in the pathogenesis among both clusters. The average menopausal age of Japanese females is around 50 years (data from the Japan Society of Obstetrics and Gynecology), and several studies have reported on the risk of developing asthma and occurrence of respiratory symptoms after menopause. ${ }^{18}$ Triebner et al. reported that menopause is a risk factor 
for the development of asthma and respiratory symptoms, independent of body mass index and age, which could be attributed to reduced estrogen levels and decreased anti-inflammatory effects inhibiting protective effects in the lungs. ${ }^{19}$ In addition, the effect of menopause on systemic inflammation has also been noted; in one study, airway inflammation in asthma patients with postmenopausal onset was significantly different from that in asthma patients with juvenile onset. ${ }^{20}$ Asthma patients with postmenopausal onset were less responsive to anti-inflammatory therapy and experienced the exacerbation more frequently. ${ }^{20}$ Based on these findings, Cluster 2 might include many postmenopausal onset cases as this group was characterized by less atopic disease type and relatively uncontrolled cases. In contrast, patients in Cluster 3 showed good symptom control and mild treatment intensity; however, they experienced exacerbations. This cluster might have experienced undertreatment, or the results might have been underestimated. Almqvist et al. ${ }^{21}$ reported on undertreatment among females, suggesting that appropriate treatment is essential for this subgroup.

In this study, non-smoking was an independent risk factor of asthma exacerbation in multivariate analysis. Although it is widely known that smoking is a risk factor for asthma exacerbation, the interpretation of this result is difficult. This study included many female patients; thus, female bias was observed in the exacerbation group. Therefore, non-smoking might have been extracted as an independent risk factor. After adjustment for sex and age, the data showed that the correlation between nonsmoking and exacerbation disappeared.

Our study has some limitations. Firstly, chronic obstructive pulmonary disease (COPD) was not excluded in this study. Therefore, COPD exacerbation might have also been included in addition to asthma exacerbation. Secondly, the physicians were mainly respiratory physicians, and it is possible that the diagnosis was biased toward severe or refractory asthma. Lastly, approximately $60 \%$ of the participants in this study were women, which could be associated with the greater possibility of nonsmokers.

In summary, we examined the factors involved in asthma exacerbation using two consecutive questionnaires for 2014 and 2015. In the multivariate analysis, there was a significant difference in transient OCS administration in the previous year, hospitalization due to asthma in the past, and smoking history, especially a nonsmoking history. Through cluster analysis, exacerbation cases were classified into three clusters: Cluster 1, slightly older cases with smoking history; Cluster 2, female-dominant, non-smoking and nonatopic cases with uncontrolled symptoms; and Cluster 3, female-dominant and non-smoking group with mild atopic cases. These results suggest the presence of several phenotypes in female patients that easily exacerbate their asthma despite a nonsmoking history and good pulmonary function. Therefore, sufficient control of symptoms is important for asthma management among female patients.

\section{Acknowledgments}

The authors are grateful for the expert help of Ms. Asako Maruyama in performing data management. This work was supported in part by Grants-in-Aid for Scientific Research from the Ministry of Education, Culture, Sports, Science and Technology of Japan (no. 15K09547).

\section{Declaration of interest}

The authors declare no conflicts of interest. The authors alone are responsible for the content and writing of this paper.

\section{References}

1. Busse W, Lemanske RF Jr. Asthma. N Engl J Med. 2001;344(5):350-62.

2. NAEPP (National Asthma Education and Prevention Program) [Internet]. Bethesda: National Heart, Lung, and Blood Institute; c2007-2017. Expert Panel Report 3: Guidelines for the Diagnosis and Management of Asthma [cited 2017 Apr 15]; [about 1 screen]. Available from: https://www.nhlbi. nih.gov/health-pro/guidelines/current/asthma-guidelines

3. Global Strategy for Asthma Management and Prevention [Internet]. Bethesda: Global Initiative for Asthma (GINA): c2016 [cited 2017 Apr 15]. Available from: http://www.ginasthma.org/.

4. Reddel HK, Taylor DR, Bateman ED, Boulet LP, Boushey HA, Busse WW, et al. An official American Thoracic Society/European Respiratory Society statement: asthma control and exacerbations: standardizing endpoints for clinical asthma trials and clinical practice. Am J Respir Crit Care Med. 2009;180(1):59-99.

5. Sato R, Tomita K, Sano H, Ichihashi H, Yamagata S, Sano A, et al. The strategy for predicting future exacerbation of asthma using a combination of the Asthma Control Test and lung function test. J Asthma. 2009;46(7): 677-82.

6. Tanaka A, Uno T, Sato H, Jinno M, Hirai K, Miyata Y, et al. Predicting future risk of exacerbations in Japanese patients with adult asthma: A prospective 1-year follow up study. Allergol Int. 2017;66(4):568-73.

7. Miller MK, Lee JH, Miller DP, Wenzel SE, Group TS. Recent asthma exacerbations: a key predictor of future exacerbations. Respir Med. 2007; 101(3):481-9.

8. Yoshimine F, Hasegawa T, Suzuki E, Terada M, Koya T, Kondoh A, et al. Contribution of aspirin-intolerant asthma to near fatal asthma based on a questionnaire survey in Niigata Prefecture, Japan. Respirology. 2005;10(4): 477-84.

9. Suzuki K, Hasegawa T, Sakagami T, Koya T, Toyabe S, Akazawa K, et al Analysis of perimenstrual asthma based on questionnaire surveys in Japan. Allergol Int. 2007;56(3):249-55.

10. Koyanagi K, Koya T, Sasagawa M, Hasegawa T, Suzuki E, Arakawa M, et al. An analysis of factors that exacerbate asthma, based on a Japanese questionnaire. Allergol Int. 2009;58(4):519-27.

11. Ota K, Hasegawa T, Koya T, Sakagami T, Sekikawa T, Toyabe S, et al. Analysis of inhaled corticosteroid selection in patients with bronchial asthma using a questionnaire survey--effects of age, gender, and disease severity. Allergol Int. 2009;58(3):365-71.

12. Sakagami T, Hasegawa T, Koya T, Furukawa T, Kawakami H, Kimura Y, et al. Cluster analysis identifies characteristic phenotypes of asthma with accelerated lung function decline. J Asthma. 2014;51(2):113-8.

13. Tsukioka K, Koya T, Ueno H, Hayashi M, Sakagami T, Hasegawa $T$, et al. Phenotypic analysis of asthma in Japanese athletes. Allergol Int. 2017;66(4):550-6.

14. Bel EH, Sousa A, Fleming L, Bush A, Chung KF, Versnel J, et al. Diagnosis and definition of severe refractory asthma: an international consensus statement from the Innovative Medicine Initiative (IMI). Thorax. 2011; 66(10):910-7.

15. Kupczyk M, ten Brinke A, Sterk PJ, Bel EH, Papi A, Chanez P, et al. Frequent exacerbators--a distinct phenotype of severe asthma. Clin Exp Allergy. 2014;44(2):212-21.

16. Awadh N, Chu S, Grunfeld A, Simpson K, FitzGerald JM. Comparison of males and females presenting with acute asthma to the emergency department. Respir Med. 1996;90(8):485-9.

17. Schatz M, Clark S, Camargo CA Jr. Sex differences in the presentation and course of asthma hospitalizations. Chest. 2006;129(1):50-5. 
18. Atwood CS, Bowen RL. A multi-hit endocrine model of intrinsic adult -onset asthma. Ageing Res Rev. 2008;7(2):114-25.

19. Triebner K, Johannessen A, Puggini L, Benediktsdottir B, Bertelsen RJ, Bifulco E, et al. Menopause as a predictor of new-onset asthma: A longitudinal Northern European population study. J Allergy Clin Immunol. 2016;137(1):50-7 e6.
20. Balzano G, Fuschillo S, De Angelis E, Gaudiosi C, Mancini A, Caputi M. Persistent airway inflammation and high exacerbation rate in asthma that starts at menopause. Monaldi Arch Chest Dis. 2007;67(3):135-41.

21. Almqvist C, Worm M, Leynaert B, working group of GALENWPG. Impact of gender on asthma in childhood and adolescence: a GA2LEN review. Allergy. 2008;63(1):47-57. 